\title{
Probing the impact of stellar duplicity on the frequency of giant planets: Final results of our VLT/NACO survey
}

\author{
Anne Eggenberger ${ }^{1}$, Stéphane Udry ${ }^{2}$, Gaël Chauvin ${ }^{1}$, \\ Thierry Forveille ${ }^{1}$, Jean-Luc Beuzit ${ }^{1}$, Anne-Marie Lagrange ${ }^{1}$ and \\ Michel Mayor ${ }^{2}$ \\ ${ }^{1}$ Université Joseph Fourier - Grenoble 1 / CNRS, Laboratoire d'Astrophysique de Grenoble \\ (UMR 5571), BP 53, 38041 Grenoble Cedex 9, France \\ email: Anne.Eggenberger@obs.ujf-grenoble.fr, Gael.Chauvin@obs.ujf-grenoble.fr, \\ Thierry.Forveille@obs.ujf-grenoble.fr, Jean-Luc.Beuzit@obs.ujf-grenoble.fr, \\ Anne-Marie. Lagrange@obs . ujf-grenoble.fr \\ ${ }^{2}$ Observatoire de Genève, Université de Genève, 51 ch. des Maillettes, 1290 Sauverny, \\ Switzerland \\ email: Stephane.Udry@unige.ch, Michel.Mayor@unige.ch
}

\begin{abstract}
If it is commonly agreed that the presence of a (moderately) close stellar companion affects the formation and the dynamical evolution of giant planets, the frequency of giant planets residing in binary systems separated by less than $100 \mathrm{AU}$ is unknown. To address this issue, we have conducted with VLT/NACO a systematic adaptive optics search for moderately close stellar companions to 130 nearby solar-type stars. According to the data from Doppler surveys, half of our targets host at least one planetary companion, while the other half show no evidence for short-period giant planets. We present here the final results of our survey, which include a new series of second-epoch measurements to test for common proper motion. The new observations confirm the physical association of two companion candidates and prove the unbound status of many others. These results strengthen our former conclusion that circumstellar giant planets are slightly less frequent in binaries with mean semimajor axes between 35 and 100 AU than in wider systems or around single stars.
\end{abstract}

Keywords. planetary systems: formation, binaries: visual, techniques: high angular resolution

\section{The NACO survey}

To probe the impact of stellar duplicity on the frequency of giant planets, we have conducted with VLT/NACO an adaptive optics search for stellar companions to $\sim 60$ planet-host stars and to $\sim 70$ non-planet-host stars (hereafter control stars). This survey revealed 95 companion candidates near 33 targets (Fig. 1). Using two-epoch astrometry we identified 19 true companions, 2 likely bound objects, and 34 background stars (Eggenberger et al. 2007). The companionship of the remaining 40 companion candidates could not be constrained due to the lack of a second-epoch astrometric measurement. Assuming that all but two of these 40 objects were unbound, we showed that giant planets seem slightly less frequent in $\sim 35-100$ AU binaries than around single stars (Eggenberger et al. 2008).

\section{New observations}

We recently performed the second-epoch measurements that were missing previously. The new observations show that the companion candidates we detected near the 

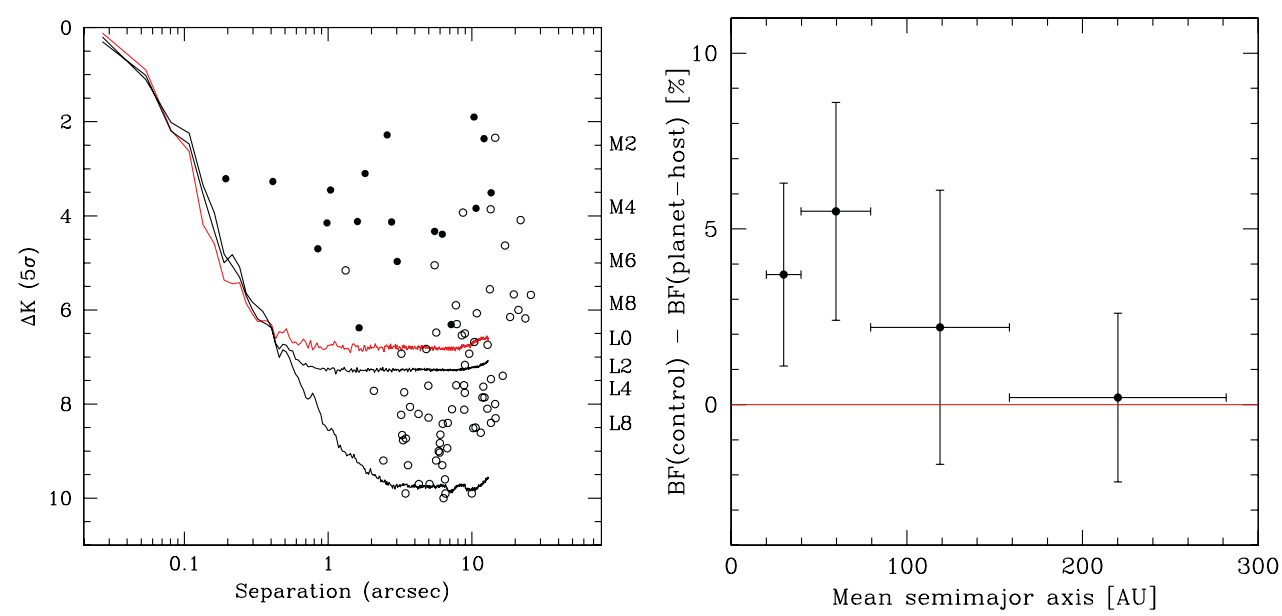

Figure 1. Results of our survey. Left: Detections and detection limits. Dots are true companions, circles are unbound objects. The uppermost curve is the detection limit used for the statistical analysis. The two lower curves are median detection limits obtained with the old and with the present detectors of NACO. Right: Difference between the binary fraction of control stars and the binary fraction of planet-host stars as a function of binary mean semimajor axis. Vertical error bars are $68 \%$ bootstrap confidence intervals. Horizontal error bars represent the bin width.

planet-host stars HD 76700, HD 83443, HD 162020, and HD 330075 are all unrelated background stars. On the other hand, the new data confirm the physical association of the companion candidates to the control stars HD 82241 and HD 134180.

\section{Statistical analysis}

Figure 1 (right) shows the difference in binary fraction between the control and the planet-host subsamples. According to the updated statistical analysis, the difference in binary fraction is $13.2 \pm 5.1 \%$ for semimajor axis below $100 \mathrm{AU}$, and $-1.5 \pm 2.9$ for semimajor axis between 100 and 200 AU. The positive difference seen for mean semimajor axis $\lesssim 100$ AU suggests that giant and intermediate-mass planets are slightly less frequent in moderately close binaries than in wider systems or around single stars. If confirmed with a larger sample, this result would support the idea that the presence of a moderately close stellar companion affects the formation of giant planets, but does not completely stop the process.

\section{Acknowledgements}

AE acknowledges support from the Swiss National Science Foundation through a fellowship for advanced researchers.

\section{References}

Eggenberger, A., Udry, S., Chauvin, G., Beuzit, J. L., Lagrange, A. M., \& Mayor, M. 2008, ASP-CS, 398, 179

Eggenberger, A., Udry, S., Chauvin, G., Beuzit, J.-L., Lagrange, A.-M., Ségransan, D., \& Mayor, M. 2007, $A \mathscr{E} A, 474,273$ 